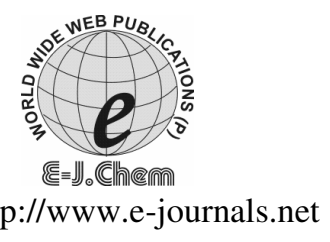

ISSN: 0973-4945; CODEN ECJHAO

E-Journal of Chemistry 2010, 7(2), 564-568

\title{
Kinetics Study of Thermal Degradation of Resin Derived from Salicylaldehyde, Ethylenediamine and Formaldehyde
}

\author{
DHANRAJ.T.MASRAM ${ }^{*}$, N.S.BHAVE ${ }^{\S}$ and K.P.KARIYA \\ *Department of Chemistry, University of Delhi, Delhi-110007, India. \\ ${ }^{\S}$ Department of Chemistry, Rashtrasant Tukadoji Maharaj Nagpur University, \\ Nagpur-440033, India. \\ Department of Chemistry, VMV commerce JMT Arts \& JJP Science College, \\ Nagpur-44000, India. \\ dhanraj_masram27@rediffmail.com
}

Received 10 September 2009; Accepted 5 November 2009

\begin{abstract}
The present paper reports the synthesis and kinetics of thermal degradation studies of resin salicylicldehyde -ethylenediamine -formaldehyde (SdEDF) derived by the condensation of salicylicldehyde and ethylenediamine with formaldehyde in the presence of catalyst hydrochloric acid in 1:1:2 molar proportions of reactants. Detailed thermal degradation studies of the SdEDF resin has been carried out to ascertain its thermal stability. Thermal degradation curve has been discussed in order to determine their mode of decomposition, order of reaction, apparent activation energy, frequency factor, free energy change, entropy change, and apparent energy change. Freeman Carroll and Sharp- Wentworth methods have been applied for the calculation of kinetic parameters while the data from the Freeman - Carroll methods have been used to determine various thermodynamic parameters.
\end{abstract}

Keywords: Freeman - Carroll, Arrhenius equation, Order of reaction, Thermal degradation.

\section{Introduction}

Terpolymers offer novelty and versatility; hence they occupy the pivotal position in the field of material science. The progress in the field terpolymers has been extremely rapid, as they generally useful in packaging, adhesives and coatings in electrical sensors and organometalic semiconductors. Some other applications have been reported in the field of activators, ion exchangers, catalyst and thermally stable materials ${ }^{1}$. Various researchers have been studied the applications of terpolymer resins of substituted phenols and formaldehyde ${ }^{2,3}$. Terpolymers of salicylic acid, thiourea with trioxane and $p$-hydroxybenzoic acid, thiourea with trioxane have been reported in the literature ${ }^{3-7}$. The present communication deals 
with synthetic and thermal degradation properties of a newly synthesized terpolymer resin derived from salicylaldehyde, ethylenediamine and formaldehyde. The Freeman-Carroll and Sharp- Wentworth methods have been applied for the calculation of kinetic parameters ${ }^{8-12}$. Methods for the estimation of kinetic parameters from thermogravimetric studies are generally based on the assumption that the Arrhenius equation is valid with thermal and diffusion barriers are negligible.

\section{Experimental}

\section{Synthesis of Salicylicldehyde - Ethylenediamine -Formaldehyde Terpolymer}

A mixture of salicylicldehyde, ethylenediamine and formaldehyde in the molar ratio 1:1:2 in $100 \mathrm{~mL}$ of $2 \mathrm{M}$ hydrochloric acid was heated in an oil bath at $120{ }^{\circ} \mathrm{C}$ for 6 hours with occasional shaking. The resinous product so obtained was repeatedly washed with distilled water dried in air and powdered with the help of agated mortar and pestle. The powder was washed many times with hot water to remove unreacted monomers. The air-dried powder was extracted with diethyl ether and then petroleum ether was used to remove salicylicldehyde - ethylenediamine and other possible copolymers, which might be present along with terpolymer. It was further purified by dissolving in $8 \%$ sodium hydroxide solution, filtered and reprecipited by gradual drop wise addition of 1:1 (v/v) hydrochloric acid with constant and rapid stirring to avoid lump formation. The SdEDF terpolymer so obtained was filtered washed several times with hot water dried and purity checked with thin layer chromatography (TLC) technique ${ }^{11,12}$.

The structure of polymer is given below (Scheme 1).

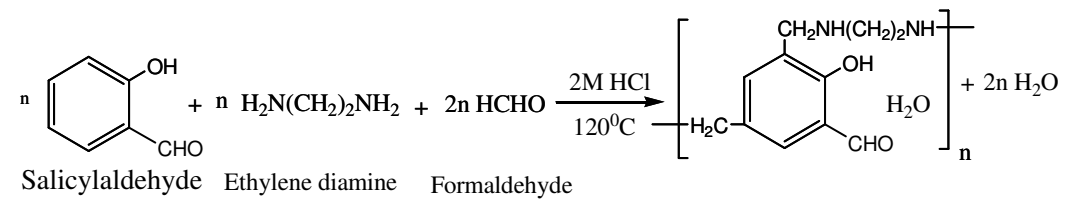

Salicylaldehyde Ethylene diamine Formaldehyde

SdEDF Terpolymer

Scheme 1.

\section{Thermogravimetry}

Thermogravimetric analyses (TGA) of terpolymer sample have been carried out by using Perkins Elmer TGS-1l thermal analyzer at heating rate of $10{ }^{\circ} \mathrm{C}$ per minute and in air atmosphere up to $800{ }^{\circ} \mathrm{C}$. The thermograms were recorded at Sophisticated Instrumentation Centre for Applied Research and Testing (SICART), Vallabh Vidyanagar, Gujrat.

Thermal analysis method is associated with a change in weight with respect to temperature. Heating is performed under strictly controlled conditions and can reveal changes in structure and other important properties of the material being studied. In nonisothermal or dynamic TGA the sample is subjected to conditions increase in temperature at linear rate ${ }^{11-13}$. The Freeman - Carroll and Sharp- Wentworth methods have been employed for the calculation of kinetic parameters of the newly synthesized terpolymer resin with help of dynamic TG curve ${ }^{11-13}$. The advantage of Freeman and Carroll method that in one single stage by keeping heating rate constant both the order of reaction and energy of activation can calculated in a single experiment. The following expression is used to evaluate various kinetic parameters:

$$
\frac{\Delta \log d w / d t}{\Delta \log W r}=n-\frac{E a}{2.303 \quad R} \cdot \frac{\Delta(1 / T)}{\Delta \log W r}
$$


Hence, a plot of $\frac{\Delta \log \left(\frac{d w}{d t}\right)}{\Delta \log W_{r}}$ vs. $\frac{\Delta(1 / T)}{\Delta \log W_{r}}$ should give a straight line with an intercept on $\mathrm{y}$-axis equal to the value of $\mathrm{n}$ (the order of reaction) and the slope $\mathrm{m}=\mathrm{Ea} /$ 2.303R.

Where, dw/dt is the rate of change of weight with time and in expression $\mathrm{Wr}=\mathrm{Wc}-\mathrm{w}$, $\mathrm{Wc}=$ weight loss at the completion of the reaction, $\mathrm{w}$ is the total weight loss up to the time $\mathrm{t}$ and $\mathrm{T}$ is the temperature in $\mathrm{k}$.

The following expression is used to evaluate Ea with Sharp- Wentworth method:

$$
\log \frac{(d c / d T)}{(1-c)}=\log (A / \beta)-\left[\frac{E_{a}}{2.303 R}\right] \cdot \frac{1}{T}
$$

Where, $d c / d t$ is the rate of change of mass with time $t, T$ is the temperature and $\beta=\Delta T / d t$.

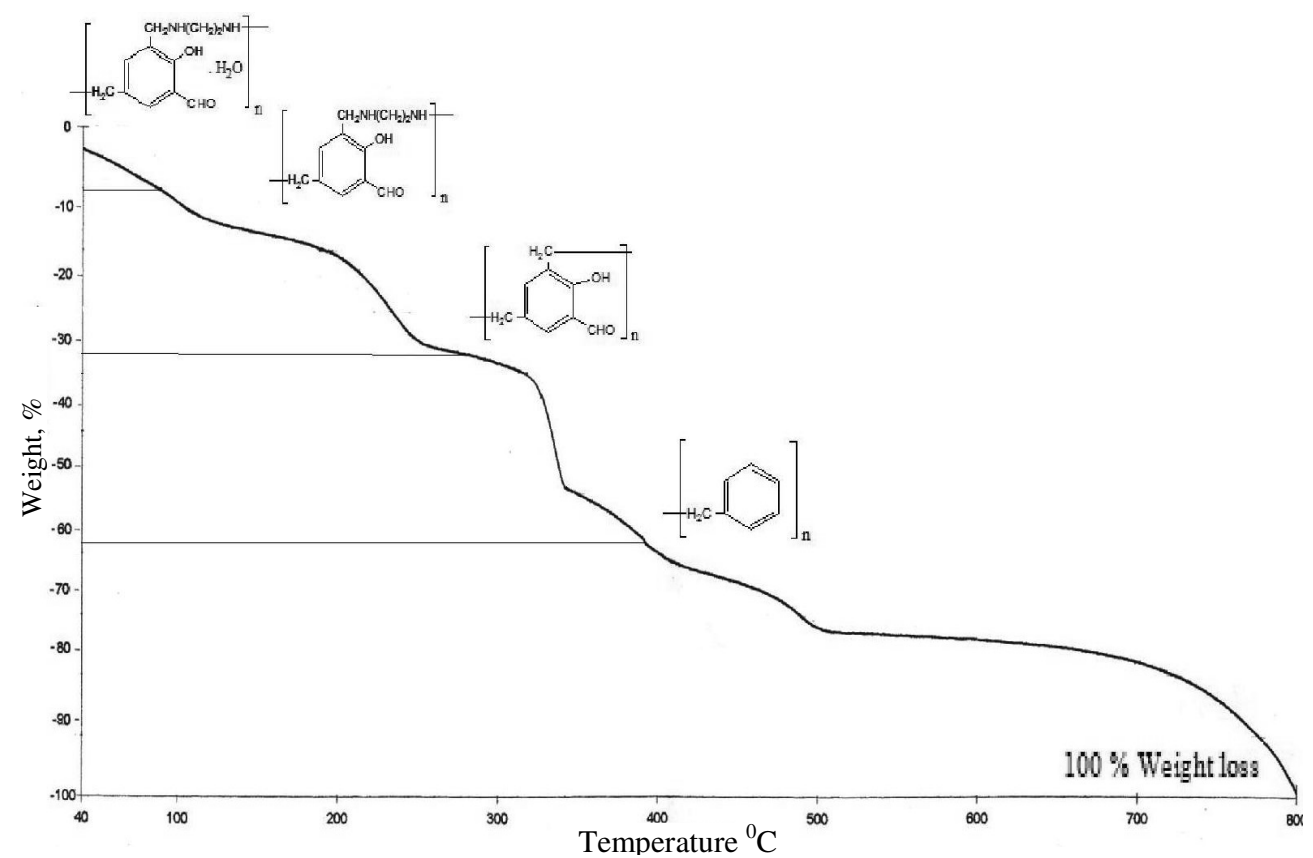

Figure 1. Thermogram of SdEDF terpolymer

\section{Results and Discussion}

The Figure 1 shows thermal degradation curve for SdEDF resin. It exhibits four-stage decomposition and ranges as per in Table 1.The first stage decomposition ranged from 40$110{ }^{\circ} \mathrm{C}$ which was slow and corresponding to loss $9.5 \%$ which may have been due to entrapped $\mathrm{H}_{2} \mathrm{O}$ molecule. The degradation of side chain attached to aromatic nucleus was found in second stage decomposition. The observed mass loss is $33.0 \%$ against calculated $33.9 \%$. The third stage decomposition at $240-340{ }^{\circ} \mathrm{C}$ is due to the loss of Loss of $-\mathrm{CH}_{2}$, $\mathrm{OH}$, and $-\mathrm{CHO}$ group observed $59.5 \%$ and calculated $60.7 \%$. The fourth state decomposition corresponds to total decomposition of resin. The Half Decomposition temperature for SdEDF resin is found to be $334^{\circ} \mathrm{C}$. 
Table 1. Thermoanalytical data and decomposition temperature of SdEDF terpolymer.

\begin{tabular}{|c|c|c|c|c|c|}
\hline \multirow{2}{*}{ Terpolymer } & \multirow{2}{*}{$\begin{array}{l}\text { Temperature } \\
\text { Range, }{ }^{\circ} \mathrm{C}\end{array}$} & \multirow{2}{*}{$\begin{array}{c}\text { Stage of } \\
\text { Decomposition }\end{array}$} & \multirow{2}{*}{ Species Degraded } & \multicolumn{2}{|c|}{$\%$ Weight loss } \\
\hline & & & & Observed & Calculated \\
\hline \multirow{4}{*}{ SdEDF } & $40-110$ & First & $\begin{array}{l}\text { Loss of entrapped }-\mathrm{H}_{2} \mathrm{O} \\
\text { molecule }\end{array}$ & 9.5 & 8.3 \\
\hline & $110-240$ & Second & $\begin{array}{l}\text { Loss of side chain attached } \\
\text { to aromatic nucleus }\end{array}$ & 33.0 & 33.9 \\
\hline & $240-340$ & Third & $\begin{array}{l}\text { Loss of }-\mathrm{CH}_{2},-\mathrm{OH} \text { and } \\
-\mathrm{CHO} \text { group. }\end{array}$ & 59.5 & 60.7 \\
\hline & $340-800$ & Fourth & Complete decomposition & 100 & 100 \\
\hline
\end{tabular}

Table 2. Result of thermogravimetric analysis of SdEDF terpolymer.

\begin{tabular}{|c|c|c|c|c|c|c|c|c|c|}
\hline \multirow[b]{2}{*}{ 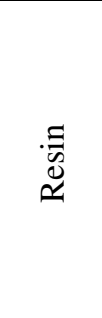 } & \multirow[b]{2}{*}{ 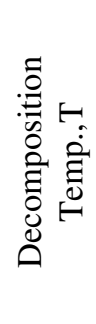 } & \multirow[b]{2}{*}{ 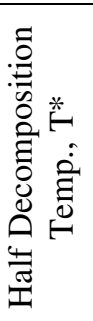 } & \multicolumn{2}{|c|}{$\begin{array}{c}\text { Activation } \\
\text { Energy } \mathrm{kJ} / \mathrm{mole}\end{array}$} & \multicolumn{5}{|c|}{ Kinetic parameters by FC } \\
\hline & & & 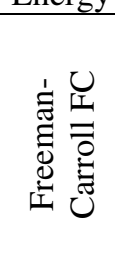 & 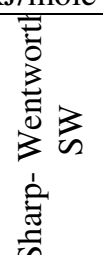 & 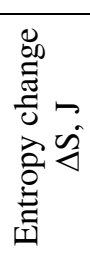 & 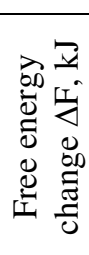 & 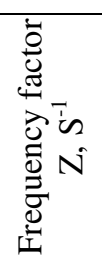 & 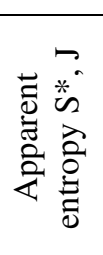 & $\mathrm{n}$ \\
\hline SdEDF & 200 & 334 & 18.09 & in & 9.26 & 15.91 & 890.64 & -22.8 & 0.98 \\
\hline
\end{tabular}

\section{Conclusion}

Thermogram of SdEDF resin shows activation energy calculated by the Freeman - Carroll and Sharp- Wentworth methods are in good agreement with each other. Thermodynamic parameters have been calculated on the basis of thermal activation energy and values are given in Table2.Due to abnormally low value of frequency factor [Z] it may be classified as a slow reaction and no other obvious reason can be given. The value of entropy $[\Delta S]$ indicates that the activated polymer has more ordered structure than the reactants and the reaction are slower than normal. This is further supported by low $\mathrm{Z}$ values ${ }^{11-15}$.It is very difficult to draw any unique conclusion from the magnitude of thermal activation energy [Ea] as decomposition mechanism is expected to be complicated. Positive values of activation energy under present investigation correspond to the energy of activation due oxidation -reduction process of terpolymer in the higher temperature range ${ }^{11-15}$.

Fairly straight-line plots are obtained using the two methods. However, using the Freeman- Carroll method some abnormal points were ignored to get a clear picture about most of the points. Similarly, in the Sharp- Wentworth method, some points at the beginning or the end did not fall on straight line. This is expected, since, the decomposition of terpolymer is not obeying first order kinetics perfectly. These observations are in harmony with the findings of Jacobs and Tompkin and other earlier workers ${ }^{15}$.

\section{Acknowledgment}

The author is thankful to the Head of the Department of Chemistry, Rashtrasant Tukadoji Maharaj Nagpur University, Nagpur for providing necessary laboratory facilities. 


\section{References}

1. Jadhao M, Paliwal L J and Bhave N S, Thermans, 2004, 250-253.

2. Das A P, Lenka S and Nayak P L, J Appl Polym Sci., 1985, 30, 4619.

3. Samal R K and Senapati B K, J Appl Polym Sci., 1996, 62, 655.

4. DeGeiso R C, Donaruma L G and Tomic E A, Anal Chem., 1962, 34, 845.

5. Michael P E, Lingala P S, Juneja H D and Paliwal L J, J Appl Polym Sci., 2004, 92, 2278.

6. Gurnule W B, Juneja H D and Paliwal L J, Asian J Chem., 1999, 11(3), 767.

7. Lingala P S, Juneja H D and Paliwal L J, Thermans 2000, 2000, 245-247.

8. Freeman E S and Carroll B, J Phys Chem., 1958, 62, 394.

9. Freeman E S and Anderson D A, J Polym Sci., 1961, 54, 253.

10. Sharp J B and Wentworth S A, Anal Chem., 1969, 41, 2060.

11. Masram D T, Kariya K P and Bhave N S, e-Polymers, 2007, 075

12. Jadhao M, Paliwal L J and Bhave N S, J Appl Polym Sci., 2005, 96,1605.

13. Coats A W and Redfen J P, Nature, 1964, 208, 68.

14. Pal T K and Kharat R B, Indian J Chem., 1989, 28A, 55-58.

15. Jacobs P W M and Tompkins F C, Chemistry of Solids State, E G, Garner Publication, London, 1955,188. 


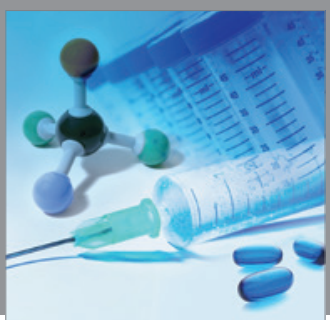

International Journal of

Medicinal Chemistry

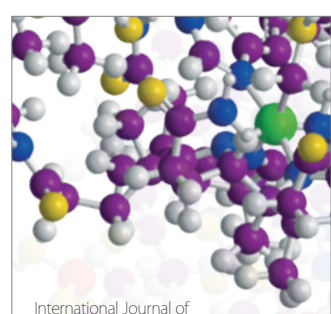

Carbohydrate Chemistry

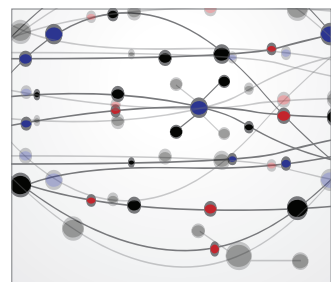

The Scientific World Journal
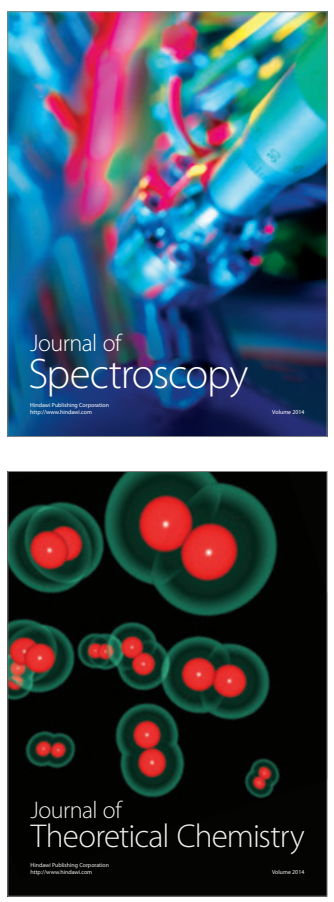
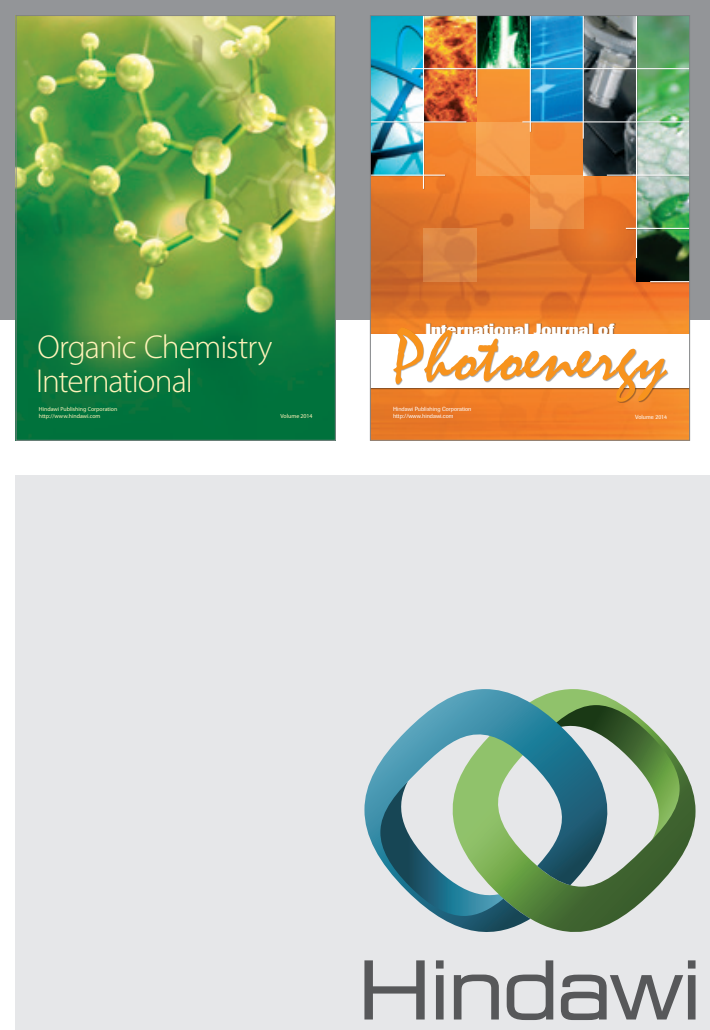

Submit your manuscripts at

http://www.hindawi.com
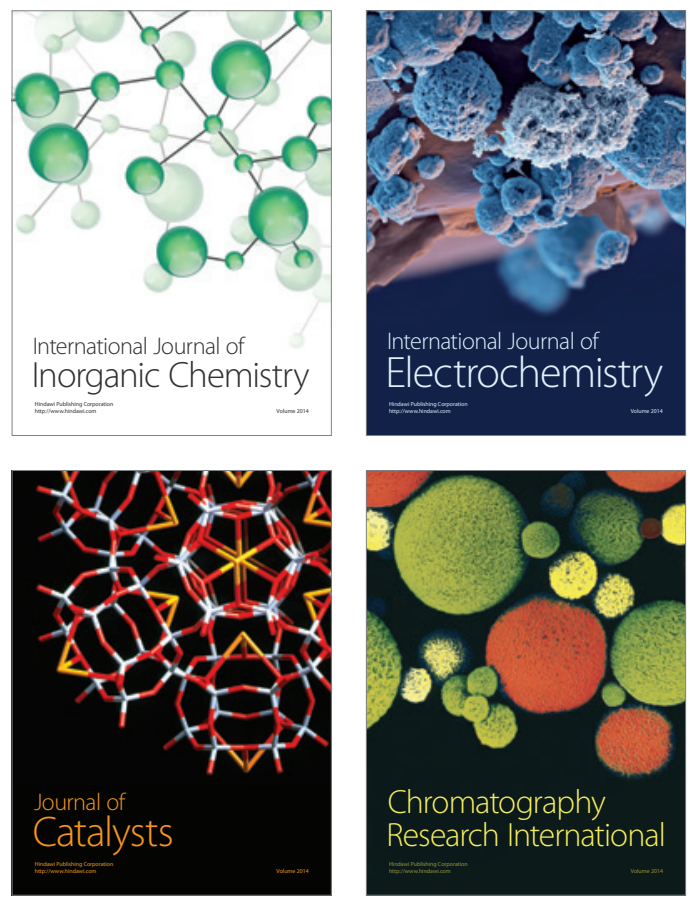
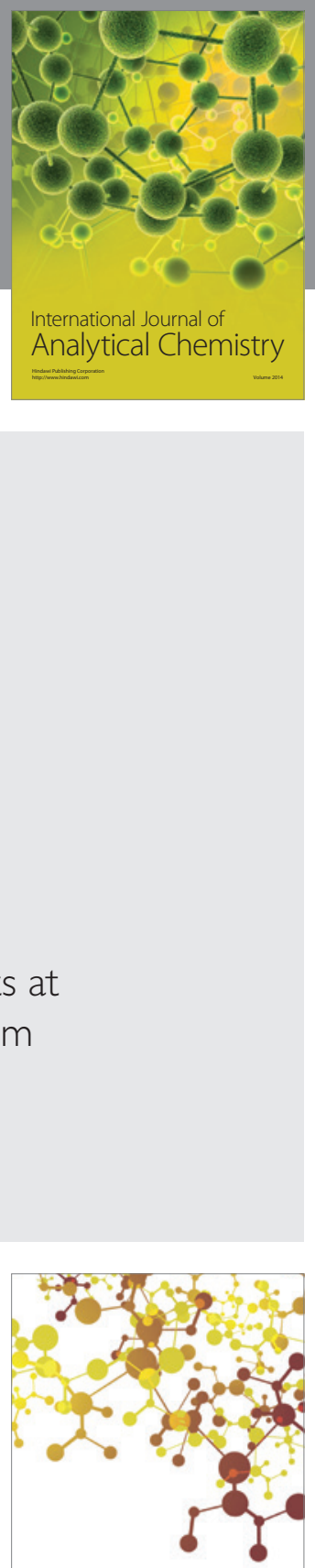

Journal of

Applied Chemistry
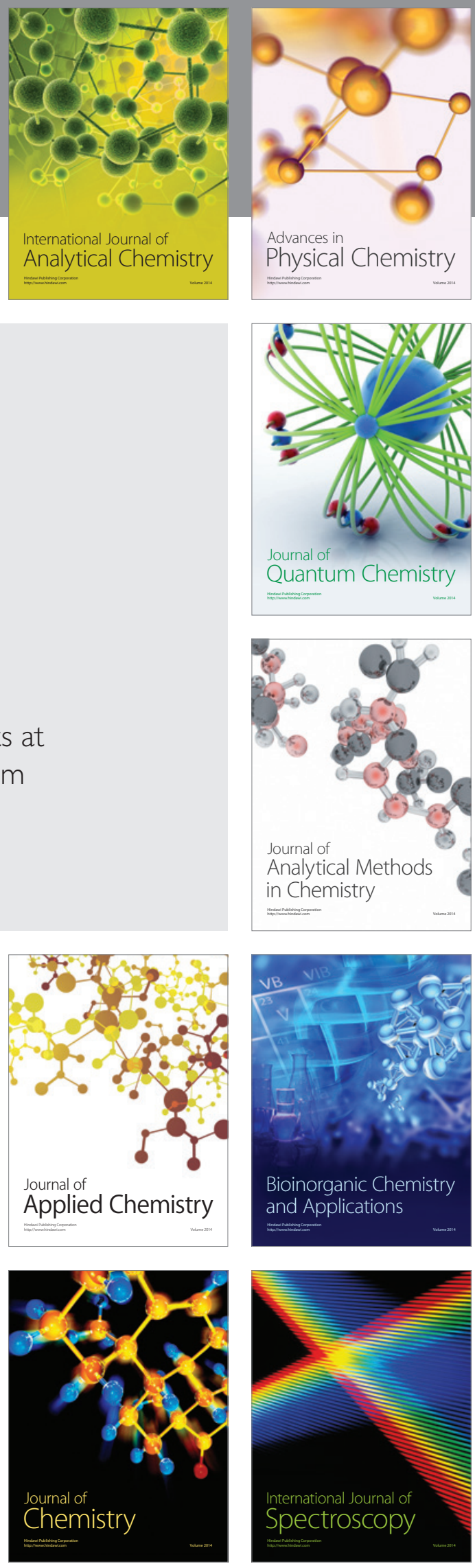